\title{
Study on the Breakdown Mechanism of Fabrication of Micro Channels in Fused Silica Substrates with ps Laser Pulses
}

\author{
S.X. LI ${ }^{a, *}$ Z.C. BAI ${ }^{b}$ AND S.J. QIN ${ }^{b}$ \\ ${ }^{a}$ School of Physics and Electronic Science, Guizhou Normal College, China \\ ${ }^{b}$ Key Lab of Photoelectron Technology and Application, University of Guizhou, China
}

(Received November 4, 2011; in final form September 29, 2012)

\begin{abstract}
A new microchannel fabrication technology for fused silica substrate is presented. A mode-locked laser was used to fabricate straight microchannels in a fused silica substrate by laser plasma-induced plasma. The depth of the channels is up to $5 \mathrm{~mm}$ and there are no thermal cracks around the channel. We studied the ionization mechanism of optical breakdown formed by laser pulses and discussed the optical breakdown threshold. A mechanism is proposed to explain the formation of the microchannels and the characteristics of the microchannels are analyzed through the laser pulse characteristics.
\end{abstract}

DOI: $10.12693 /$ APhysPolA.123.67

PACS: 52.50.Jm, 52.40.Hf, 61.80.-x, 42.62.-b

\section{Introduction}

The fabrication technology of micro/nano channel is now hot, which is used in many fields such as microelectronic mechanical system (MEMS), DNA, and blood analysis. Traditional micro-fabrication methods of microchannels contain electron beam lithography, reactive ion etching, masking et al. But these methods have shortcomings, such as expensive and time consuming [1-3]. Much attention has been paid to laser micromachining because of a number of advantages: single-step processing with high flexibility, and no contamination of material being processed etc. [1-4]. The channels of a few $\mu \mathrm{m}$ in diameter with depth over $1 \mathrm{~mm}$ have been produced in quartz with fs, ps and ns laser pulses [4]. There are many cracks around the channel. The method of laser-induced plasma assisted-ablation using a Nd:YAG (1064 nm) laser proposed by Qin and $\mathrm{Li}[1-3]$ is quick and non-polluting. The depth of the micro channel is up to $4 \mathrm{~mm}$ and the diameter is $25-200 \mu \mathrm{m}$ which can be controlled by setting the dimension of the drilling pattern. In these papers, the channels were fabricated from the front surface of the sample and only the process of microchannels were described qualitatively, without putting forward the specific theoretical model.

Interaction between the laser and the materials is complex. When the laser radiation is incident on the materials, various phenomena will occur, including reflection, refraction, absorption, scattering, and transmission. The most important phenomena in the laser processing of materials is the absorption of laser energy, which results in various effects, such as, heating, melting, vaporization, plasma formation etc. Many scholars [1-6] research laser damages and processing in the different materials because

*corresponding author; e-mail: lishixiong1985@126.com of its special properties and applications. Plasma formation plays an important role during the interaction between laser and transparent materials. It is necessary for sufficiently larger laser intensity.

To analyze the plasma formation and expansion during laser materials interaction, many researchers $[7-17]$ put forward many theories and models, such as ionization mechanism, moving breakdown model, laser-supported shock wave etc. During the laser-materials interaction, the intense laser pulse ionizes the materials and produces an optical breakdown, even plasma spark, which can be characterized by a high density of free electrons $N_{\mathrm{cr}}=10^{18} \mathrm{~cm}^{-3}$ [12]. The rate equations of electrons can be used to calculate breakdown thresholds according to the multiphoton ionization and cascade ionization $[7,11-17]$. Based on the breakdown threshold of optical breakdown, a moving breakdown model of interactions between laser pulses and materials were proposed by Raizer [7] and modified by Docchio et al. and Fan et al. $[8,9]$. It is assumed that optical breakdown will take place as soon as the incoming laser intensity exceeds the threshold value. Plasma formation is accompanied by the generation of a shock wave, which increased plasma processing. The model of the laser-supported shock wave generated by plasma expansion was researched using hydrodynamic equations [10].

As reported by Qin and $\mathrm{Li}[1-3]$, there are two methods to fabricate microchannel in quartz: thermal induced processing and laser-induced plasma processing. Since the energy of the laser was not high enough to melt the quartz from the exit surface, the channel was formed primarily through thermal cracking. This is called thermal-induced processing and there are many thermal cracks around the channel. If the quartz was first pre-damaged at a site by thermal-induced processing, and the laser beam focused on this site on the entrance surface, then plasma can be produced from the site of the entrance sur- 
face once the laser intensity reached the sufficient value. A channel with smooth internal surface was fabricated by the melt of plasma. This process is called laser-induced plasma processing. In this paper, a different method was used to fabricate microchannels in a fused silica substrate by laser plasma-induced plasma. The channels fabricated by this method are longer and much smaller than those mentioned in [1-4]. The formation mechanisms and characteristic of microchannels will be analyzed.

\section{Experiments}

\subsection{Experimental apparatus}

There are two experimental apparatuses in our work, the microscope system and the laser system. The laser system used in the experiment is depicted in Fig. 1. A mode-locked laser, with wavelength $\lambda=355 \mathrm{~nm}$, was used in the laser system. The frequency of the laser pulse was $80 \mathrm{MHz}$ and the pulse width of the laser was $15 \mathrm{ps}$. The maximum laser power radiation on the sample was $10 \mathrm{~W}(0.125 \mu \mathrm{J}$ pulse energy $)$. The laser beam was first expanded by the beam expander and focused by a scanning lens with a focal length of $330 \mathrm{~mm}$ onto the sample surface. The Gaussian spot size of the beam was determined to be around $400 \mu \mathrm{m}^{2}$ at $355 \mathrm{~nm}$ (radius of $12 \mu \mathrm{m}$ at $1 / \mathrm{e}^{2}$ intensity, radius of the focal spot $r \approx \lambda f / \pi a$, where $f$ is focal length and $a$ is the radius of the beam at the focal lens). The experiments were conducted in air with atmospheric pressure and the microscope system is fluorescence microscope BM-13 (Shanghai Shangguang Industry Co., Ltd).

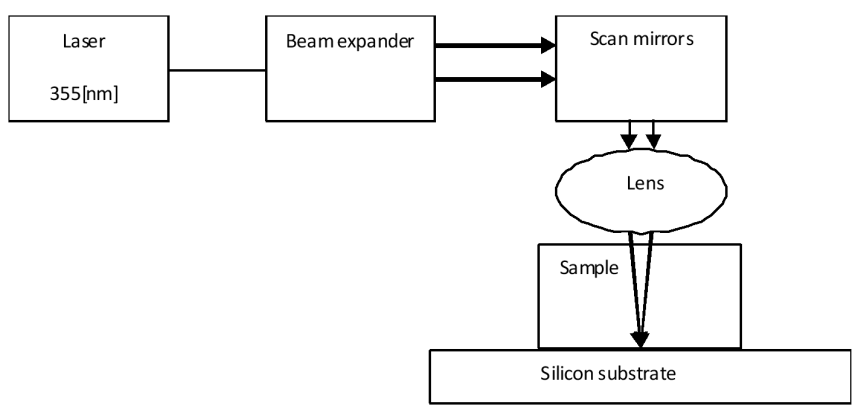

Fig. 1. The experimental setup for fabricating of microchannels. It contains a mode-locked laser (Newport, USA), beam expander, scan mirrors and lens (China Lasers (Wuhan) Co., Ltd), fused silica $\left(20 \times 20 \times 10 \mathrm{~mm}^{3}\right.$ Shanghai Optical Class Co., Ltd), silicon substrate $\left(50 \times 50 \times 2 \mathrm{~mm}^{3}\right.$, Shanghai Jiujing Electronic Materials, Inc).

We have used different methods of laser irradiation to process fused silica. (1) The laser beam was directly focused on the entrance and exit surface of fused silica. (2) The fused silica substrate was first pre-damaged at a site by thermal-induced processing and the laser beam was focused on this damaged site. The exit surface of fused silica was in the air for (1) and (2). (3) The fused silica was directly placed on the surface of silicon substrate and the laser beam focused on the exit surface of fused silica (or surface of silicon substrate) to process (see Fig. 1).

\subsection{Experimental results}

As to the methods (1) and (2), we cannot obtain any damage except hot fused silica. But for the third method, we observed the plasma spark, then a microchannel was formed. As the laser beam was focused on the exit surface of fused silica, plasma was first formed on the silicon surface. After a few seconds (or less than one second), plasma was produced at laser focus area in fused silica substrates accompanying strong plasma spark. The spark time is less than one second, with the continuous laser irradiation; the number of plasma spark is no more than 3 times, only 1-2 times.

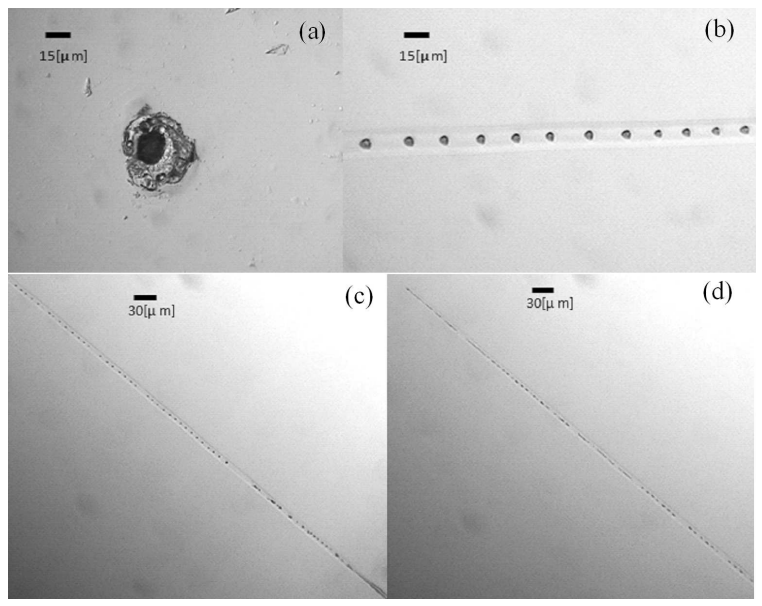

Fig. 2. Microscope photos of microchannels drilled by laser plasma-induced plasma with the mode-locked laser (10 W power, $15 \mathrm{ps}$ duration) after $1-5 \mathrm{~s}$ irradiation. (a) The surface entrance of channel and (b), (c), (d), respectively, express the kerf surface of the middle part of channel, the beginning part of channel, and the end part of the channel.

A microchannel of high quality was produced after plasma sparks. This is called laser plasma-induced plasma. Microscope photos of a microchannel fabricated by laser plasma-induced plasma are shown in Fig. 2. Figure $2 \mathrm{a}$ is the surface entrance of channel. There is some damage around the entrance of channel. Figure $2 b-d$, respectively, expresses the kerf surface of the middle part of channel, the front part of channel and the end part of the channel. The depth of the microchannel is up to $5 \mathrm{~mm}$ and the diameter of microchannel is $10 \mu \mathrm{m}$. The channel fabricated by this process is of high quality with a uniform diameter from the entrance to the end part of the channel and no thermal cracks around the channel are observed, as shown in Fig. 2. It is worth noting that part of the channel is not connected. There are series of damaged cavities in the channel, and these hollows are 
regularly arranged in the channel with the approximate same interval as shown in Fig. 2b-d.

\section{Theoretical analysis}

\subsection{Mechanism of processing}

There is a major difficulty in using a laser to process fused silica. The fused silica is an optically transparent material and its average absorptivity for the spectrum from visible to near ultraviolet is less than $5 \%$. The physical principle that allowed us to process optically transparent fused silica is the so-called electric breakdown effect of the electric field [18]. Based on the effect, the electric field strength at the exit surface can be higher than the electric field strength at the laser beam entrance surface. It is easier to process fused silica from the rear surface of substrate using thermal-induced processing, but there are many thermal cracks around the channels. In our work, the channels were fabricated by laser plasma-induced plasma processing from the exit surface. It is a consequence of the rapid energy deposition into medium by laser pulses.

Plasma was first formed on the silicon surface because of the high absorption rate of the laser, so the fused silica could be melted and gasified by the plasma. The following laser pulses can be easily absorbed by the materials because of the increasing energy absorption rate, so the gas can easily absorb the following laser pulses and can be easily ionized. The rapid ionization of the gas leads to a high temperature and pressure plasma with the electron density exceeding a critical value $N_{\mathrm{cr}}=10^{18} \mathrm{~cm}^{-3}$, which is called optical breakdown accompanied by a plasma spark and sharp sound. The plasma attacked and melted the neighboring materials and expanded because of the absorption of subsequent laser energy. The plasma expansion leads to a laser-supported shock wave and produces a great pressure on the surface of material. This leads to rapid attacking and melting of the neighboring fused silica, which in turn gives rise to a rapid energy transfer from the laser pulses to the material. The plasma functions forward as a piston as long as the plasma can be maintained by the subsequent laser energy. Thus the plasma could produce a microchannel followed by the expansion of plasma and the emission of shock wave.

\subsection{Distribution of laser intensity}

The Gaussian temporal profile of a laser pulse takes the form $[8,9]$ :

$$
P(t)=P_{\max } \exp \left((-4 \ln 2)\left(\frac{t}{\tau_{\mathrm{p}}}\right)^{2}\right),
$$

where $P_{\max }$ is the peak power of the laser pulse, and $\tau_{\mathrm{p}}$ is the full width at half maximum (FWHM) pulse duration. If $T_{0}$ is the half width at half maximum (HWHM) pulse duration, then $\tau_{\mathrm{p}}=1.665 T_{0}$. The peak power and pulse energy has the relation of $E_{\mathrm{p}}=1.064 P_{\max } \tau_{\mathrm{p}}$.

When the laser beam is focused on a small spot, the beam spot size $w$ is a function of axial position $z$, which can be characterized by $[9,19]$ :

$$
w(z)=w_{0}\left(1+\frac{z^{2}}{z_{\mathrm{R}}^{2}}\right)^{1 / 2},
$$

where $z_{\mathrm{R}}=n_{0} \pi w_{0}^{2} / \lambda$ is the Rayleigh length, $n_{0}$ is the refractive index, $\lambda$ is wavelength and $w_{0}$ is beam waist.

The intensity of a Gaussian beam falls off very rapidly with radius beyond the spot size $w$. The radial intensity variation of a Gaussian beam with spot size $w$ is given by [19]:

$$
I(r, z)=\frac{2 P}{\pi w^{2}(z)} \exp \left(-\frac{2 r^{2}}{w^{2}(z)}\right),
$$

where $P$ is power of laser pulse, $r$ is the radius beyond the beam centre. Combining the temporal pulse Eq. (1) and the position-dependent intensity in Eq. (3), the pulse irradiance $I(r, z, t)$ both time and position-dependent can be expressed as

$$
\begin{aligned}
& I(r, z, t)=\frac{2 P_{\max } \exp \left((-4 \ln 2)\left(t / \tau_{\mathrm{p}}\right)^{2}\right)}{\pi w^{2}(z)} \\
& \quad \times \exp \left(-\frac{2 r^{2}}{w^{2}(z)}\right) .
\end{aligned}
$$

Equation (4) represents the laser intensity which is both time and position dependent. Let us note $z=0$, $r=0$, represents the center of the beam focus and $t=0$ corresponds to the pulse peak $P_{\max }$ arriving at the point. That is to say the maximum laser intensity is at the beam focus for the time $t=0$.

\subsection{Mechanisms of optical breakdown}

High-power laser beam focused on the optical materials can lead to optical breakdown, resulting from the formation of plasma. Plasma formation in optical breakdown is the result of the ionization. There are two mechanisms which can lead to plasma: direct ionization of the medium by multiphoton ionization and cascade ionization.

Multiphoton ionization: in the case that the ionization energy of the atom is much higher than the energy of the laser quanta, the electron can break away from the atom directly by the laser beam, as a result of a simultaneous absorption of many photons. The minimum number of photons for multiphoton ionization is $[14,16]$ :

$$
k=\operatorname{int}\left(1+\frac{\Delta E}{\hbar \omega}\right) .
$$

Here $\Delta E$ is the ionization energy, $\hbar=h /(2 \pi)$, and $h$ is the Planck constant, $\omega$ is angular frequency of laser. The symbol $\operatorname{int}(x)$ denotes the integer part of the number $x$. For the fixed ionization energy, the number $k$ depends on the photon energy (or laser wavelength). As an example, for the fused silica $\left(\mathrm{SiO}_{2}, \Delta E=9 \mathrm{eV}\right)$ and laser wavelength of $\lambda=355 \mathrm{~nm}$, if a free electron can be generated by multiphoton ionization, the number of absorbed photons is $k=3$.

According to Keldysh's expression for multiphoton ionization, the multiphoton ionization rate in condensed media is given by $[14,16]$ : 


$$
\begin{aligned}
& \left(\frac{\mathrm{d} N}{\mathrm{~d} t}\right)_{\mathrm{mp}}=\frac{2 \omega}{9 \pi}\left(\frac{m^{\prime} \omega}{\hbar}\right)^{3 / 2} \\
& \quad \times \exp (2 k) \Phi\left(\sqrt{2 k-\frac{2 \Delta E}{\hbar \omega}}\right) \\
& \quad \times\left(\frac{e^{2}}{16 m^{\prime} \Delta E \omega^{2} c \varepsilon_{0} n_{0}} I\right)^{k} .
\end{aligned}
$$

Here $m^{\prime}$ is reduced exciton mass $\left(m^{\prime} \approx m / 2\right), N$ is the free-electron density as a function of time $t$ and axial position $z, I$ is the laser intensity, $k$ is the minimum number of photons for multiphoton ionization determined by Eq. (5), $n_{0}$ is the refractive index, $c$ is the velocity of light in free space, $\varepsilon_{0}$ is the permittivity of free space, $\Phi(x)$ represents Dawson's integral

$$
\Phi(x)=\exp \left(-x^{2}\right) \int_{0}^{x} \exp \left(y^{2}\right) \mathrm{d} y
$$

The multiphoton ionization rate gives the number of free electrons generated per unit time per unit volume. It is as a function of the input irradiance $I$. Using Eqs. (4) and (6) we can calculate the free electron density generated by multiphoton ionization during the $\Delta t$.

Cascade ionization: once seed electrons are generated in the focal volume, they gain energy from the electric field through inverse bremsstrahlung absorption and are accelerated. If the electron accumulates sufficient energy for ionization, the electron ionizes the atom (or molecule), so that one electron is replaced by two slow ones. As the same process repeats, more and more electrons can be produced just as the avalanche effect. The avalanche ionization rate is given by $[14,15]$ :

$$
\eta=\frac{\tau}{\omega^{2} \tau^{2}+1}\left(\frac{e^{2} I}{n_{0} c \varepsilon_{0} m \Delta E}-\frac{m \omega^{2}}{M}\right),
$$

where $M$ is the atomic (or molecular) mass, $n_{0}$ is the refractive index, $\varepsilon_{0}$ is the permittivity of free space, $c$ is the vacuum speed of light, $e$ and $m$ is the charge and mass of an electron, $\tau$ is the mean free time between collisions.

For optical breakdown, in addition to multiphoton ionization and avalanche ionization, it also contains the electronic diffusion and electron-ion recombination loss. The generic form of such a rate equation is $[11,12,15]$ :

$$
\frac{\mathrm{d} N}{\mathrm{~d} t}=\left(\frac{\mathrm{d} N}{\mathrm{~d} t}\right)_{\mathrm{mp}}+\eta N-g N,
$$

where $N$ is the electron density, $(\mathrm{d} N / \mathrm{d} t)_{\mathrm{mp}}$ is electron generation of multiphoton ionization, $\eta$ is electron generation rate of cascade ionization. The first two terms represent a gain of electron density. The remaining terms refer to the electron losses due to diffusion and recombination. Let us note that the multiphoton ionization and cascade ionization are a function of laser intensity. It is both time and position dependent. We can calculate the electron density and discuss the optical breakdown by the breakdown threshold.

\subsection{Results and discussion}

Many researchers have used rate equations of electrons to calculate breakdown thresholds according to the multi- photon ionization and cascade ionization in gases, liquids and solids [11, 13, 15, 17]. With different laser parameters, most previous investigations have either neglected multiphoton ionization or electron losses [11, 12, 15], and focused on the breakdown thresholds. For ultrashort pulses, multiphoton ionization is the dominating mechanism, because at the high irradiance values required for breakdown of the multiphoton ionization rate is much higher than the cascade ionization rate. For a long laser pulse, multiphoton ionization may still contribute to the generation of the initial free electrons. Once the cascade ionization occurs, the free electron density formed by cascade ionization will be greater than the electron density formed by multiphoton ionization.

Here we first calculated the free electron density generated only by the multiphoton ionization by Eq. (6) (10 W power). From the above analysis, note that the input irradiance $I$ as a function of time and position, setting $r=0, z=0$ and $t=0$, we can obtain approximate maximum free electron density generated during pulse duration $\tau_{\mathrm{p}}$. The maximum free electron density is $N_{\text {max }}=3 \times 10^{11} \mathrm{~cm}^{-3}$, it is far less than optical breakdown threshold $N_{\mathrm{c}}=10^{18} \mathrm{~cm}^{-3}$. During the laser pulse, multiphoton ionization cannot lead to optical breakdown because of the weak laser intensity. In fact, the free electrons generated during pulse duration $\tau_{\mathrm{p}}$ cannot reach the maximum density $N_{\max }$, because the pulse is the Gauss type, rather than a rectangular pulse. Laser intensity is not always maintained in the maximum intensity during pulse duration. In addition, because of the free electron losses, multiphoton ionization cannot produce the maximum free electron density $N_{\max }$. But multiphoton ionization can provide initial electrons for avalanche ionization.

Once the seed electrons are produced, electron cascade can occur in the high laser field. Here, neglecting the electron losses and multiphoton ionization, Eq. (9) can be expressed by

$$
\frac{\mathrm{d} N}{\mathrm{~d} t}=\eta N,
$$

note that the laser pulse is Gaussian type rather than rectangular pulse, $\eta$ is not constant, solution of the above equation is

$$
N=N_{0} \exp \left(\int_{0}^{t} \eta \mathrm{d} t\right),
$$

where $N_{0}$ is the initial value of electron density. Combining Eq. (4), Eq. (8) and Eq. (11), the free electron density generated during pulse duration $\tau_{\mathrm{p}}$ can be calculated. In view of approximate treatment, we used the peak laser intensity (setting $r=0, z=0$ and $t=0$, Eq. (4) is equal to $I(0,0,0))$, combining Eq. (8), and obtained the maximum avalanche ionization rate (fused silica) $\eta_{\max }=5 \times 10^{10}$. When the pulse duration $\tau_{\mathrm{p}}$ is $15 \times 10^{-12} \mathrm{~s}$, the free electron density $N=2 N_{0}$. Apparently, the relation between avalanche ionization coefficient and pulse width plays an important role in accumulation of free electron produced by avalanche ionization. If the product of avalanche ion- 
ization coefficient and pulse width is greater than 10 , avalanche ionization phenomenon will be obvious.

As to above calculation, the product of $\eta$ and $t$ is less than 1 , and the number of free electrons produced by avalanche ionization equal the initial electrons. That is to say, avalanche ionization cannot cause optical breakdown. For long pulse, avalanche ionization plays an important role in optical breakdown. For example, we used the pulse with $1 \mathrm{~ns}$, and assumed $\eta_{\max }=5 \times 10^{10}$, then $N=5 N_{0} \times 10^{21}$. If the initial electron density is $N_{0}=10^{11} \mathrm{~cm}^{-3}$, the electron density $N$ will be far greater than the optical breakdown threshold. This also agrees with other scholars' demonstration that for long pulse, cascade ionization is the dominating mechanism.

In our work, we used a mode-locked laser with a pulse width $\tau_{\mathrm{p}}=15 \times 10^{-12} \mathrm{~s}$ at wavelength $\lambda=355 \mathrm{~nm}$ to make microchannels in fused silica substrates. From above analysis, if we consider the multiphoton ionization, avalanche ionization, and even the position of maximum laser intensity, it cannot accumulate enough free electrons to form the optical breakdown and the plasma spark. In our experiments, the laser beam was directly focused on the entrance and exit surface of fused silica, but we did not observe any plasma spark, and there was no observed damage. It also verified the correctness of above theory.

We know that silicon plasma is easily formed because of the high absorption rate. When the fused silica was directly placed on silicon substrates and the laser beam was focused on the exit surface of fused silica, plasma was first formed on the silicon surface and the formation of the damage around the entrance of channel is due to the ablation of the silicon plasma. After a few seconds (or less than one second), plasma was produced at the laser focus area in fused silica substrates accompanying strong plasma spark.

The relaxation time between silicon plasma formation and fused silica plasma formation is called energy deposition time. During the energy deposition time, the fused silica around the plasma can be melted and vaporized, increasing the energy absorption rate of fused silica. So the fused silica can be ionized rapidly by the absorption of laser energy. Once the electron density reached optical breakdown threshold, the plasma of fused silica was formed. The plasma attacked and melted the neighboring media and formed the new plasma with the absorption of subsequent laser energy. With the following laser pulses, plasma expanded against the direction of the laser propagation in the form of a shock wave. So the laser plasma-induced plasma of fused silica was produced to drill a microchannel.

The microscope photos of microchannels fabricated by laser plasma-induced plasma and thermal induced processing are shown in Fig. 3. As shown in Fig. 3b, there are many thermal cracks around the channel fabricated by thermal induced processing. The thermal cracks were formed mainly through thermal stresses. As shown in Fig. 3a, the channel fabricated by laser plasma-induced plasma is of high quality and no thermal cracks are ob- served. For this process, the laser-induced plasma of fused silica was used to perform drilling by melting, and thermal cracks can be eliminated by melting.

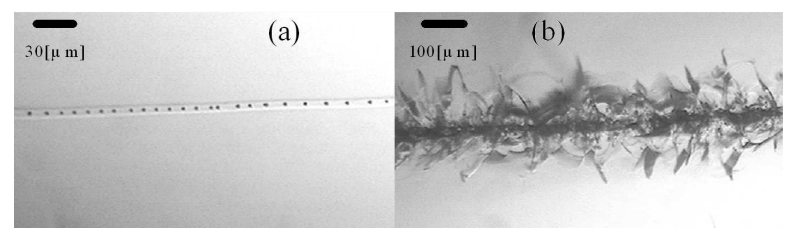

Fig. 3. Microscope photos of microchannel for (a) microchannel drilled by laser plasma-induced plasma with ps laser pulses and (b) microchannel drilled by thermal induced processing with ns laser pulses (laser power is $3.5 \mathrm{~W}$, pulse width is $140 \mathrm{~ns}$, China Lasers (Wuhan) Co., Ltd).

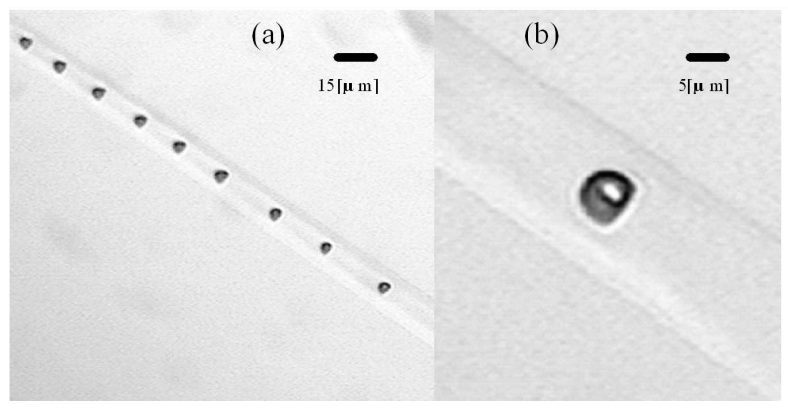

Fig. 4. Microscope photos of microchannel drilled by laser plasma-induced plasma processing for (a) damage points distributed in equal interval and (b) a damage spot in the channel.

Figure 4 is microscope photos of the microchannel drilled by laser plasma-induced plasma processing. There are series of damage spots in the channel, the hollows are regularly arranged in the channel with the approximate same interval. This is due to the pulse characteristics of mode-locked laser. The pulse is Gaussian type and the laser pulse irradiated to the media with the same interval. At the middle of the pulse, the laser intensity is strongest and formed the strong plasma which can lead to a hollow. Then the plasma absorbed the pulses energy with the same interval and ablated the regular hollows.

\section{Conclusion}

Experimental study of fabrication of microchannels in fused silica substrates by laser plasma-induced plasma is presented. The technology not only allows the rapid construction of channel but also creates high quality microchannels with a uniform size and the depth of the channels is up to $5 \mathrm{~mm}$. We analyzed the process of microchannel formation and mainly studied the mechanisms of optical breakdown by cascade ionization and multiphoton ionization, and analyzed the reason of plasma formation. It is a consequence of the rapid energy deposition 
into medium by laser pulses. The plasma formation not only relates to laser density but also depends on the energy deposition (or energy absorption rate). The theoretical analysis agrees with the experimental result well. The ablation of high temperature and pressure plasma led to a microchannel of high quality with a smooth internal surface and no thermal cracks. The new technology will lead to new applications of MEMS in biomedical engineering.

\section{References}

[1] S.J. Qin, W.J. Li, Appl. Phys. A 74, 773 (2002)

[2] S.J. Qin, W.J. Li, Sensors Actuators A 97-98, 749 (2002)

[3] S.J. Qin, W.J. Li, Acta Mech. Sin. 20, 125 (2004)

[4] H. Varel, D. Ashkenasi, A. Rosenfeld, M. Wahmer, E.E.B. Campbell, Appl. Phys. A 65, 367 (1997).

[5] M. Srecković, J. Ilić, M. Davidović, B. Djokić, Ž. Tomić, Z. Latinović, D. Družijanić, Acta Phys. Pol. A 116, 618 (2009).

[6] M. Srećković, J. Ilić, A. Kovacević, S. Pantelić, Z. Lationović, N. Borna, V. Cosović, Acta Phys. Pol. A 112, 935 (2007).

[7] Yu.P. Raizer, Sov. Phys. Usp. 8, 650 (1966)
[8] F. Docchio, P. Regondi, M.R.C. Capon, J. Mellerio, Appl. Opt. 27, 3661 (1988).

[9] C.H. Fan, J.P. Longtin, Appl. Opt. 40, 3124 (2001)

[10] A.N. Pirri, Phys. Fluids 16, 1435 (1973)

[11] Y.R. Shen, The Principles of Nonlinear Optics Wiley, New York 1984.

[12] N. Bloembergen, IEEE J. Quant. Electron. QE-10, 375 (1974)

[13] C. DeMichelis, IEEE J. Quant. Electron. QE-5, 188 (1969)

[14] J. Noack, A. Vogel, IEEE J. Quant. Electr. 35, 1156 (1999).

[15] P.K. Kennedy, IEEE J. Quant. Electron. 31, 2241 (1995)

[16] L.V. Keldysh, Sov. Phys.-JETP 20, 1307 (1965).

[17] C.H. Fan, J. Sun, J.P. Longtin, J. Appl. Phys. 91, 2530 (2002)

[18] C.J. Nonhof, Material Processing with Nd-Lasers, Electrochemical Publications, Ayr, UK 1988.

[19] A.E. Siegman, Lasers, University Science Books, Sausalito, CA 1986. 